Moroccan J. of Pure and Appl. Anal. (MJPAA)

Volume 6(1), 2020, Pages 1-15

ISSN: Online 2351-8227 - Print 2605-6364

DOI: $10.2478 / \mathrm{mjpaa}-2020-0001$

\title{
Limit cycles of Liénard polynomial systems type by averaging method
}

\author{
Amel Boulfoul $^{1}$ and Nawal Mellahi ${ }^{2}$
}

Aвstract. We apply the averaging theory of first and second order for studying the limit cycles of generalized polynomial Linard systems of the form

$$
\dot{x}=y-l(x) y, \quad \dot{y}=-x-f(x)-g(x) y-h(x) y^{2},
$$

where $l(x)=\epsilon l_{1}(x)+\epsilon^{2} l_{2}(x), f(x)=\epsilon f_{1}(x)+\epsilon^{2} f_{2}(x), g(x)=\epsilon g_{1}(x)+\epsilon^{2} g_{2}(x)$ and $h(x)=\epsilon h_{1}(x)+\epsilon^{2} h_{2}(x)$ where $l_{k}(x)$ has degree $m$ and $f_{k}(x), g_{k}(x)$ and $h_{k}(x)$ have degree $n$ for each $k=1,2$, and $\epsilon$ is a small parameter.

Mathematics Subject Classification (2010): 34C29, 34C25, 47H11.

Key words and phrases: Limit cycle, Linard differential system, Periodic orbit, Averaging theory.

\section{Introduction and statement of the main results}

The second part of the $16^{\text {th }}$ Hilbert's problem [14] wants to find an uniform upper bound for the number of limit cycles of all polynomial differential systems of a given degree. The $16^{\text {th }}$ problem, except the one related with the Riemann hypothesis, seems to be the most elusive of Hilbert's problems.

Following to Smale [28] many authors consider an easier and special class of polynomial differential systems, the Linard polynomial differential systems

$$
\dot{x}=y, \dot{y}=-x-f(x) y,
$$

Received : 27 November 2019 - Accepted: 25 February 2020.

(C) This article is published with open access by Sidi Mohamed Ben Abdallah University.

The Author(s): 1,2 Department of mathematics; 20 august 1955 University, BP26; El Hadaiek 21000, Skikda. Algeria e-mail: ${ }^{1}$ boulfoul_amel@yahoo.fr

e-mail: ${ }^{2}$ nawel.mellahi@yahoo.fr . 
where $f(x)$ is a polynomial in the variable $x$ of degree $n$.

In 1977 Lins et al. [17] stated the conjecture that if $f(x)$ has degree $n \geq 1$ then system (1.1) has at most $[n / 2]$ limit cycles. They proved this conjecture for $n=1,2$. The conjecture for $n=3$ has been proved by Chengzi and Llibre in [15]. For $n \geq 5$ the conjecture is not true, see De Maesschalck and Dumortier [7] and Dumortier et al. [10]. So it remains to know if the conjecture is true or not for $n=4$.

The following class of polynomial differential systems

$$
\dot{x}=y, \quad \dot{y}=-g(x)-f(x) y,
$$

where $f(x)$ and $g(x)$ are polynomials in the variable $x$ of degrees $n$ and $m$, respectively is a generalisation of the Linard polynomial differential systems (1.1).

Many results on limit cycles of polynomial differential systems have been obtained by considering limit cycles which bifurcate from a single degenerate singular point, that are so called small amplitude limit cycles, see [22]. We denote by $\hat{H}(m, n)$ the maximum number of small amplitude limit cycles for systems of the form (1.2). The values of $\hat{H}(m, n)$ give a lower bound for the maximum number $\mathrm{H}(\mathrm{m}, \mathrm{n})$ (i.e. the Hilbert number) of limit cycles that the differential equation (1.2) with $\mathrm{m}$ and $\mathrm{n}$ fixed can have.

- In 1928 Linard [16] proved that if $\mathrm{m}=1$ and $F(x)=\int_{0}^{x} f(s) d s$ is a continuous odd function, which has a unique root at $\mathrm{x}=\mathrm{a}$ and is monotone increasing for $x \geq a$, then equations (1.2) has a unique limit cycle.

- In 1973, Rychkov [26] proved that if $\mathrm{m}=1$ and $F(x)$ is an odd polynomial of degree five, then equations (1.2) have at most two limit cycles.

- In 1977, Lins et al. [17] proved that $H(1,1)=0$ and $H(1,2)=1$.

- Dumortier et al. in [11] and [8] proved that $H(3,1)=1$.

- In 1997, Dumortier and Li [9] proved that $H(2,2)=1$.

- In 1998 Coppel [6] proved that $\mathrm{H}(2,1)=1$.

Blows, Lloyd [2] and Lynch ([23],[24]) have used inductive arguments in order to prove the following results:

- If $g$ is odd then $\hat{H}(m, n)=[n / 2]$.

- If $f$ is even then $\hat{H}(m, n)=n$, whatever $g$ is.

- If $f$ is odd then $\hat{H}(m, 2 n+1)=[(m-2) / 2]+n$.

- If $g(x)=x+g_{e}(x)$, where $g_{e}$ is even then $\hat{H}(2 m, 2)=m$.

Christopher and Lynch [5] developed a new algebraic method for determining the Liapunov quantities of system (1.2) and proved the following:

- $\hat{H}(m, 2)=[(2 m+1) / 3]$.

- $\hat{H}(2, n)=[(2 n+1) / 3]$.

- $\hat{H}(m, 3)=2[(3 m+2) / 8]$ for all $1<m \leq 50$.

- $\hat{H}(3, n)=2[(3 n+2) / 8]$ for all $1<n \leq 50$.

- $\hat{H}(4, k)-\hat{H}(k, 4)$ for $k=6,7,8,9$ and $\hat{H}(5,6)=\hat{H}(6,5)$.

In 1998 Gasull and Torregrosa [13] obtained upper bounds for $\hat{H}(7,6), \hat{H}(6,7), \hat{H}(7,7)$, and $\hat{H}(4,20)$. In $2006 \mathrm{Yu}$ and Han in [30] give some accurate values of $\hat{H}(m, n)=\hat{H}(n, m)$ for $n=4, m=10,11,12,13 ; n=5, m=6,7,8,9 ; n=6, m=5,6$. 
There are many results using the averaging theory studying the maximum number of limit cycles $\tilde{H}(m, n)$ which can bifurcate from the periodic orbits of a linear center $\dot{x}=y, \dot{y}=-x$, perturbed inside the class of generalized polynomial Linard differential systems:

- In 2010, Llibre et al. [18] compute the maximum number of limit cycles $\tilde{H}_{k}(m, n)$ of systems (1.2), using the averaging theory of order $k$, for $k=1,2,3$.

- In 2012, Llibre and Valls [21] studied using the averaging theory of first and second order the following systems

$$
\dot{x}=y-g_{1}(x)-f_{1}(x) y, \dot{y}=-x-g_{2}(x)-f_{2}(x) y,
$$

where $g_{1}(x), f_{1}(x), g_{2}(x)$ and $f_{2}(x)$ have degree $k, l, m$ and $n$, respectively.

- In 2013, Llibre and Valls studied using the averaging theory of third order the following systems (see [19] and [20] respectively)

$$
\begin{aligned}
& \dot{x}=y-g_{1}(x), \dot{y}=-x-g_{2}(x)-f_{2}(x) y, \\
& \dot{x}=y-f_{1}(x) y, \dot{y}=-x-g_{2}(x)-f_{2}(x) y,
\end{aligned}
$$

where $g_{1}(x), f_{1}(x), g_{2}(x)$ and $f_{2}(x)$ have degree $k, l, m$ and $n$, respectively.

- In 2016, using the averaging theory of first and second order, Mellahi et al. [25] studied the maximum number of limit cycles bifurcating from the periodic orbits of the linear center, $\dot{x}=-y, \dot{y}=x$ perturbed inside a class of generalized Kukles polynomial differential systems

$$
\left\{\begin{array}{l}
\dot{x}=-y+l(x), \\
\dot{y}=x-f(x)-g(x) y-h(x) y^{2}-d_{0} y^{3} .
\end{array}\right.
$$

- In 2019, Boulfoul et al. [3] introduced the more generalized Kukles systems

$$
\left\{\begin{array}{l}
\dot{x}=-y, \\
\dot{y}=x-f(x)-g(x) y-h(x) y^{2}-l(x) y^{3} .
\end{array}\right.
$$

- In 2014, García et al. [12] studied the maximum number of small amplitude limit cycles for the more generalized polynomial Linard differential systems

$$
\dot{x}=y, \quad \dot{y}=-x-f(x)-g(x) y-h(x) y^{2},
$$

where $f(x), g(x)$, and $h(x)$ have degree $n$.

In this paper using the averaging theory of first and second order, we study the maximum number of limit cycles which bifurcate from the periodic orbits of the linear center $\dot{x}=y, \dot{y}=$ $-x$, perturbed inside the class of the more generalized polynomial Linard differential systems

$$
\left\{\begin{array}{l}
\dot{x}=y-l(x) y, \\
\dot{y}=-x-f(x)-g(x) y-h(x) y^{2},
\end{array}\right.
$$

where $l(x)=\epsilon l_{1}(x)+\epsilon^{2} l_{2}(x), f(x)=\epsilon f_{1}(x)+\epsilon^{2} f_{2}(x), g(x)=\epsilon g_{1}(x)+\epsilon^{2} g_{2}(x)$ and $h(x)=$ $\epsilon h_{1}(x)+\epsilon^{2} h_{2}(x)$ where $l_{k}(x)$ has degree $m, f_{k}(x), g_{k}(x)$ and $h_{k}(x)$ have degree $n$ for each $k=1,2$, and $\epsilon$ is a small parameter.

Note that when $l(x)=h(x)=0$, coincides with generalized polynomial Linard differential 
systems (1.2).

In what follows we present our main results.

Theorem 1. For $|\epsilon|>0$ sufficiently small, the maximum number of limit cycles of the generalized polynomial Linard differential systems (1.4) bifurcating from the periodic orbits of linear center $\dot{x}=y$, $\dot{y}=-x$, using the averaging theory of first order is

$$
\tilde{H}_{1}(m, n)=\left[\frac{n}{2}\right]
$$

limit cycles. Moreover, this upper bound is reached.

Section 3 contains the proof of Theorem 1.

Theorem 2. For $|\epsilon|>0$ sufficiently small, the maximum number of limit cycles of the generalized polynomial Linard differential systems (1.4) bifurcating from the periodic orbits of linear center $\dot{x}=$ $y, \dot{y}=-x$, using the averaging theory of second order is

$$
\tilde{H}_{2}(m, n)=\max \left\{n,\left[\frac{n-1}{2}\right]+\left[\frac{m-1}{2}\right]+1\right\}
$$

limit cycles.

Section 4 contains the proof of Theorem 2.

In [12], it has been shown that there exist generalized Linard equation (1.3), having at least $n$ limit cycles. The result in Theorem 2 improves this lower estimate $\left(\tilde{H}_{2}(m, n)>n\right.$ for all $n \geq 3$ and $m>n+2$ ).

Note that we do not know if the upper bound $\tilde{H}_{2}(m, n)$ for the number of limit cycles of the polynomial differential system (1.4), which bifurcate from the periodic solutions of the linear center $\dot{x}=y, \dot{y}=-x$ using the averaging theory of second order is reached. We prove that the upper bound is reached for $1 \leq n \leq 5$ and $1 \leq m \leq 5$ in the second order averaging theory (see Example in Section 4).

\section{Averaging theory of first and second order}

We consider the differential system

$$
\dot{x}=\epsilon F_{1}(t, x)+\epsilon^{2} F_{2}(t, x)+\epsilon^{3} R(t, x, \epsilon),
$$

where $F_{1}, F_{2}: \mathbb{R} \times D \rightarrow \mathbb{R}, R: \mathbb{R} \times D \times\left(-\epsilon_{f}, \epsilon_{f}\right) \rightarrow \mathbb{R}$ are continuous functions, T-periodic in the first variable, and $D$ is an open subset of $\mathbb{R}$. Assume that the following hypotheses (i), (ii) hold.

(i): $F_{1}(t,.) \in C^{2}(D), F_{2}(t,.) \in C^{1}(D)$ for all $t \in \mathbb{R}, F_{1}, F_{2}, R$ are locally Lipschitz with respect to $x$, and $R$ is twice differentiable with respect to $\epsilon$. 
We define $F_{k 0}: D \rightarrow \mathbb{R}$ for $k=1,2$ as

$$
\begin{aligned}
& F_{10}(z)=\frac{1}{T} \int_{0}^{T} F_{1}(s, z) d s, \\
& F_{20}(z)=\frac{1}{T} \int_{0}^{T}\left[D_{z} F_{1}(s, z) \cdot y_{1}(s, z)+F_{2}(s, z)\right] d s,
\end{aligned}
$$

where

$$
y_{1}(s, z)=\int_{0}^{s} F_{1}(t, z) d t
$$

(ii): For $V \subset D$ an open and bounded set and for each $\epsilon \in\left(-\epsilon_{f}, \epsilon_{f}\right) \backslash\{0\}$, there exists $a \in V$ such that $F_{10}(a)+\epsilon F_{20}(a)=0$ and $d_{B}\left(F_{10}+\epsilon F_{20}, V, a\right) \neq 0$. The expression $d_{B}\left(F_{10}+\right.$ $\left.\epsilon F_{20}, V, a\right) \neq 0$ means that the Brouwer degree of the function $F_{10}+\epsilon F_{20}: V \rightarrow \mathbb{R}$ at the fixed point $a$ is not zero. A sufficient condition for the inequality to be true is that the Jacobian of the function $F_{10}+\epsilon F_{20}$ at $a$ is not zero.

Then, for $|\epsilon|>0$ sufficiently small there exists a T-periodic solution $\varphi(., \epsilon)$ of the equation (2.1) such that $\varphi(0, \epsilon) \rightarrow a$ when $\epsilon \rightarrow 0$.

- If $F_{10}$ is not identically zero, then the zeros of $F_{10}+\epsilon F_{20}$ are mainly the zeros of $F_{10}$ for $\epsilon$ sufficiently small. In this case the previous result provides the averaging theory of first order. - If $F_{10}$ is identically zero and $F_{20}$ is not identically zero, then the zeros of $F_{10}+\epsilon F_{20}$ are mainly the zeros of $F_{20}$ for $\epsilon$ sufficiently small. In this case the previous result provides the averaging theory of second order.

For a general introduction to averaging theory see ([27], [4], [29]).

\section{Proof of Theorem 1}

This proof is based on the first order averaging theory, by using the coordinates $(\theta, r)$ with

$$
x=r \cos \theta, y=r \sin \theta \text { and } r>0 .
$$

In this context, we take

$$
f_{1}(x)=\sum_{i=0}^{n} a_{i} x^{i}, \quad g_{1}(x)=\sum_{i=0}^{n} b_{i} x^{i}, \quad h_{1}(x)=\sum_{i=0}^{n} c_{i} x^{i} \text { and } l_{1}(x)=\sum_{i=0}^{m} e_{i} x^{i},
$$

then system (1.4) with $\mathrm{k}=1$ can be written as

$$
\left\{\begin{array}{l}
\dot{r}=-\epsilon\left(\sum_{i=0}^{n} P_{i, 1}(\theta, r) r^{i}+\sum_{i=0}^{m} e_{i} R_{i+1}(\theta) r^{i+1}\right), \\
\dot{\theta}=-1-\frac{\epsilon}{r}\left(\sum_{i=0}^{n} Q_{i, 1}(\theta, r) r^{i}-\sum_{i=0}^{m} e_{i} T_{i}(\theta) r^{i+1}\right),
\end{array}\right.
$$

where

$$
\begin{aligned}
& P_{i, 1}(\theta, r)=a_{i} R_{i}(\theta)+b_{i} T_{i}(\theta) r+c_{i} S_{i}(\theta) r^{2}, \\
& R_{j}(\theta)=\cos ^{j} \theta \sin \theta, \\
& T_{j}(\theta)=\cos ^{j} \theta \sin ^{2} \theta=\cos ^{j} \theta-\cos ^{j+2} \theta,
\end{aligned}
$$




$$
\begin{aligned}
& S_{j}(\theta)=\cos ^{j} \theta \sin ^{3} \theta=\cos ^{j} \theta \sin \theta-\cos ^{j+2} \theta \sin \theta, \\
& Q_{i, 1}(\theta, r)=a_{i} \cos ^{i+1} \theta+b_{i} R_{i+1}(\theta) r+c_{i} T_{i+1}(\theta) r^{2} .
\end{aligned}
$$

Now taking $\theta$ as the new independent variable, system (3.3) becomes

$$
\frac{d r}{d \theta}=\frac{\dot{r}}{\dot{\theta}}=\epsilon F_{1}(\theta, r)+O\left(\epsilon^{2}\right)
$$

where

$$
F_{1}(\theta, r)=\sum_{i=0}^{n} P_{i, 1}(\theta, r) r^{i}+\sum_{i=0}^{m} e_{i} R_{i+1}(\theta) r^{i+1}
$$

Hence

$$
F_{10}(r)=\frac{1}{2 \pi}\left(\sum_{i=0}^{n} r^{i}\left(a_{i} J_{i}(2 \pi)+b_{i} r \tilde{I}_{i}(2 \pi)+c_{i} r^{2} \tilde{J}_{i}(2 \pi)\right)+\sum_{i=0}^{m} e_{i} J_{i+1}(2 \pi) r^{i+1}\right)
$$

where

$$
J_{k}(2 \pi)=\int_{0}^{2 \pi} R_{k}(\theta) d \theta, \tilde{I}_{k}(2 \pi)=\int_{0}^{2 \pi} T_{k}(\theta) d \theta, \tilde{J}_{k}(2 \pi)=\int_{0}^{2 \pi} S_{k}(\theta) d \theta .
$$

Now using the expressions of the integrals in appendix A, we obtain

$$
F_{10}(r)=r \sum_{i=0}^{\left[\frac{n}{2}\right]} r^{2 i} b_{2 i} \tilde{I}_{2 i}(2 \pi)=r \sum_{i=0}^{\left[\frac{n}{2}\right]} r^{2 i} \frac{\alpha_{i}}{2^{i+1}(i+1) !} b_{2 i}
$$

where

$\alpha_{k}=3.5 \ldots . .(2 k-1), \alpha_{k+1}=(2 k+1) \alpha_{k}$.

Then the polynomial $F_{10}(r)$ has at most $\left[\frac{n}{2}\right]$ positive roots. We can choose the coefficients $b_{2 i}$ and, in addition, these coefficients appear multiplied by nonzero constants, it is possible to reach this upper bound. Hence, Theorem 1 is proved.

\section{Proof of Theorem 2}

As before we apply the change of variables (3.1). If we write $f_{1}(x), g_{1}(x), h_{1}(x)$ and $l_{1}(x)$ as in (3.2), and

$$
f_{2}(x)=\sum_{i=0}^{n} p_{i} x^{i}, \quad g_{2}(x)=\sum_{i=0}^{n} q_{i} x^{i}, \quad h_{2}(x)=\sum_{i=0}^{n} s_{i} x^{i} \text { and } l_{2}(x)=\sum_{i=0}^{m} v_{i} x^{i},
$$


then system (1.4) with $k=1,2$ takes the form

$$
\left\{\begin{aligned}
\dot{r} & =-\epsilon\left(\sum_{i=0}^{n} P_{i, 1}(\theta, r) r^{i}+\sum_{i=0}^{m} e_{i} R_{i+1}(\theta) r^{i+1}\right)-\epsilon^{2}\left(\sum_{i=0}^{n} P_{i, 2}(\theta, r) r^{i}\right. \\
& \left.+\sum_{i=0}^{m} v_{i} R_{i+1}(\theta) r^{i+1}\right), \\
\dot{\theta} & =-1-\frac{\epsilon}{r}\left(\sum_{i=0}^{n} Q_{i, 1}(\theta, r) r^{i}-\sum_{i=0}^{m} e_{i} T_{i}(\theta) r^{i+1}\right)-\frac{\epsilon^{2}}{r}\left(\sum_{i=0}^{n} Q_{i, 2}(\theta, r) r^{i}\right. \\
& \left.-\sum_{i=0}^{m} v_{i} T_{i}(\theta) r^{i+1}\right),
\end{aligned}\right.
$$

where

$P_{i, 2}(\theta, r)=p_{i} R_{i}(\theta)+q_{i} T_{i}(\theta) r+s_{i} S_{i}(\theta) r^{2}$,

$Q_{i, 2}(\theta, r)=p_{i} \cos ^{i+1} \theta+q_{i} R_{i+1}(\theta) r+s_{i} T_{i+1}(\theta) r^{2}$.

Taking $\theta$ as the new independent variable, system (4.1) becomes

$$
\frac{d r}{d \theta}=\frac{\dot{r}}{\dot{\theta}}=\epsilon F_{1}(\theta, r)+\epsilon^{2} F_{2}(\theta, r)+O\left(\epsilon^{3}\right)
$$

where

$$
\begin{aligned}
F_{1}(\theta, r)= & \sum_{i=0}^{n} P_{i, 1}(\theta, r) r^{i}+\sum_{i=0}^{m} e_{i} R_{i+1}(\theta) r^{i+1}, \\
F_{2}(\theta, r)= & \sum_{i=0}^{n} P_{i, 2}(\theta, r) r^{i}+\sum_{i=0}^{m} v_{i} R_{i+1}(\theta) r^{i+1}-\frac{1}{r}\left(\sum_{i=0}^{n} P_{i, 1}(\theta, r) r^{i}\right. \\
& \left.+\sum_{i=0}^{m} e_{i} R_{i+1}(\theta) r^{i+1}\right)\left(\sum_{i=0}^{n} Q_{i, 1}(\theta, r) r^{i}-\sum_{i=0}^{m} e_{i} T_{i}(\theta) r^{i+1}\right) .
\end{aligned}
$$

To compute $F_{20}(r)$, we need that $F_{10}(r)$ be identically zero. Then from (3.6) we consider

$$
b_{2 i}=0, \quad \forall 0 \leq i \leq\left[\frac{n}{2}\right] .
$$

First, using (4.5) and, by substituting in (4.3) we obtain

$$
F_{1}(\theta, r)=\sum_{i=0}^{n} r^{i}\left(a_{i} R_{i}(\theta)+c_{i} r^{2} S_{i}(\theta)\right)+\sum_{i=0}^{\left[\frac{n-1}{2}\right]} r^{2 i+2} b_{2 i+1} T_{2 i+1}(\theta)+\sum_{i=0}^{m} e_{i} r^{i+1} R_{i+1}(\theta) .
$$

Then

$$
\begin{aligned}
\frac{d F_{1}(\theta, r)}{d r}= & \sum_{i=0}^{n} r^{i-1}\left(i a_{i} R_{i}(\theta)+(i+2) c_{i} r^{2} S_{i}(\theta)\right)+\sum_{i=0}^{\left[\frac{n-1}{2}\right]}(2 i+2) b_{2 i+1} r^{2 i+1} T_{2 i+1}(\theta) \\
& +\sum_{i=0}^{m}(i+1) e_{i} r^{i} R_{i+1}(\theta) .
\end{aligned}
$$


Again, using the integrals of appendix A, we obtain

$$
\begin{aligned}
y(\theta, r)= & \int_{0}^{\theta} F_{1}(s, r) d s \\
= & \sum_{i=0}^{n} r^{i}\left(a_{i} J_{i}(\theta)+c_{i} r^{2} \tilde{J}_{i}(\theta)\right)+\sum_{i=0}^{\left[\frac{n-1}{2}\right]} r^{2 i+2} b_{2 i+1} \sum_{l=0}^{i+1} \tilde{\gamma}_{i, l} \sin ((2 l+1) \theta) \\
& +\sum_{i=0}^{m} e_{i} r^{i+1} J_{i+1}(\theta),
\end{aligned}
$$

where

$$
\tilde{\gamma}_{i, l}= \begin{cases}\gamma_{i, l}-\gamma_{i+1, l}, & 0 \leq l \leq i, \\ -\gamma_{i+1, i+1}, & l=i+1\end{cases}
$$

Now, we determine the corresponding function

$$
F_{20}(r)=F_{20}^{1}(r)+F_{20}^{2}(r),
$$

with

$$
\begin{aligned}
& F_{20}^{1}(r)=\frac{1}{2 \pi} \int_{0}^{2 \pi} \frac{d F_{1}(\theta, r)}{d r} \cdot y(\theta, r) d \theta, \\
& F_{20}^{2}(r)=\frac{1}{2 \pi} \int_{0}^{2 \pi} F_{2}(\theta, r) d \theta .
\end{aligned}
$$

In the following Lemmas we obtain some results of the integrals $F_{20}^{1}(r)$ and $F_{20}^{2}(r)$.

Lemma 3. The integral $F_{20}^{1}(r)$ is a polynomial in the variable $r$ is

$$
\begin{aligned}
F_{20}^{1}(r)= & \sum_{s=0}^{\left[\frac{n}{2}\right]} \sum_{k=0}^{\left[\frac{n-1}{2}\right]} r^{2 s+2 k+1}\left(a_{2 s} b_{2 k+1}\left(s \sum_{l=0}^{k+1} \tilde{\gamma}_{k, l} C_{s, l}-\frac{(k+1) \alpha_{k+s+1}}{2^{k+s+1}(2 s+1)(k+s+2) !}\right)\right. \\
& \left.+c_{2 s} b_{2 k+1} r^{2}\left((s+1) \sum_{l=0}^{k+1} \tilde{\gamma}_{k, l} K_{s, l}-\frac{(k+1)(4 k+10 s+15) \alpha_{k+s+1}}{2^{k+s+2}(2 s+1)(2 s+3)(k+s+3) !}\right)\right) \\
& +\sum_{k=0}^{\left[\frac{n-1}{2}\right]} \sum_{s=0}^{\left[\frac{m-1}{2}\right]} e_{2 s+1} b_{2 k+1} r^{2 s+2 k+3}\left(\frac{-(k+1) \alpha_{k+s+2}}{2^{k+s+2}(2 s+3)(k+s+3) !}+(s+1)\right. \\
& \left.\times \sum_{l=0}^{k+1} \tilde{\gamma}_{k, l} C_{s+1, l}\right) .
\end{aligned}
$$

Proof. From (4.6) and (4.7) we have

$$
\begin{aligned}
F_{20}^{1}(r)= & \sum_{i=0}^{n} \sum_{j=0}^{n} r^{i+j-1}\left(i a_{i} a_{j} \int_{0}^{2 \pi} R_{i}(\theta) J_{j}(\theta) d \theta+i a_{i} c_{j} r^{2} \int_{0}^{2 \pi} R_{i}(\theta) \tilde{J}_{j}(\theta) d \theta\right. \\
& \left.+(i+2) c_{i} a_{j} r^{2} \int_{0}^{2 \pi} S_{i}(\theta) J_{j}(\theta) d \theta+(i+2) c_{i} c_{j} r^{4} \int_{0}^{2 \pi} S_{i}(\theta) \tilde{J}_{j}(\theta) d \theta\right)
\end{aligned}
$$




$$
\begin{aligned}
& +\sum_{i=0}^{n} \sum_{k=0}^{\left[\frac{n-1}{2}\right]} r^{i+2 k+1}\left(a _ { i } b _ { 2 k + 1 } \left(i \sum_{l=0}^{k+1} \tilde{\gamma}_{k, l} \int_{0}^{2 \pi} R_{i}(\theta) \sin ((2 l+1) \theta) d \theta+(2 k+2)\right.\right. \\
& \left.\times \int_{0}^{2 \pi} T_{2 k+1}(\theta) J_{i}(\theta) d \theta\right)+c_{i} b_{2 k+1} r^{2}\left((i+2) \sum_{l=0}^{k+1} \tilde{\gamma}_{k, l} \int_{0}^{2 \pi} S_{i}(\theta) \sin ((2 l+1) \theta) d \theta\right. \\
& \left.\left.+(2 k+2) \int_{0}^{2 \pi} T_{2 k+1}(\theta) \tilde{J}_{i}(\theta) d \theta\right)\right)+\sum_{i=0}^{n} \sum_{j=0}^{m} r^{i+j}\left(a _ { i } e _ { j } \left(i \int_{0}^{2 \pi} R_{i}(\theta) J_{j+1}(\theta) d \theta\right.\right. \\
& \left.+(j+1) \int_{0}^{2 \pi} R_{j+1}(\theta) J_{i}(\theta) d \theta\right)+c_{i} e_{j} r^{2}\left((i+2) \int_{0}^{2 \pi} S_{i}(\theta) J_{j+1}(\theta) d \theta\right. \\
& \left.\left.+(j+1) \int_{0}^{2 \pi} R_{j+1}(\theta) \tilde{J}_{i}(\theta) d \theta\right)\right)+\sum_{s=0}^{\left[\frac{n-1}{2}\right]\left[\frac{n-1}{2}\right]} \sum_{k=0}^{2 k}(2 k+2) b_{2 s+1} b_{2 k+1} r^{2 s+2 k+3} \\
& \times \sum_{l=0}^{s+1} \tilde{\gamma}_{s, l} \int_{0}^{2 \pi} T_{2 k+1}(\theta) \sin ((2 l+1) \theta) d \theta+\sum_{k=0}^{\left[\frac{n-1}{2}\right]} \sum_{i=0}^{m} e_{i} b_{2 k+1} r^{i+2 k+2}((2 k+2) \\
& \left.\times \int_{0}^{2 \pi} T_{2 k+1}(\theta) J_{i+1}(\theta) d \theta+(i+1) \sum_{l=0}^{k+1} \tilde{\gamma}_{k, l} \int_{0}^{2 \pi} R_{i+1}(\theta) \sin ((2 l+1) \theta) d \theta\right) \\
& +\sum_{i=0}^{m} \sum_{j=0}^{m}(j+1) e_{i} e_{j} r^{i+j+1} \int_{0}^{2 \pi} R_{j+1}(\theta) J_{i+1}(\theta) d \theta .
\end{aligned}
$$

For simplifying expression of the polynomial $F_{20}^{1}(r)$, using the integrals of appendix A we have

- $\int_{0}^{2 \pi} R_{i}(\theta) J_{j}(\theta) d \theta=\int_{0}^{2 \pi} R_{i}(\theta) \tilde{J}_{j}(\theta) d \theta=\int_{0}^{2 \pi} S_{i}(\theta) J_{j}(\theta) d \theta=$

$\int_{0}^{2 \pi} S_{i}(\theta) \tilde{J}_{j}(\theta) d \theta=\int_{0}^{2 \pi} T_{i}(\theta) \sin (2 l+1) \theta d \theta=0$.

- $\int_{0}^{2 \pi} T_{2 k+1}(\theta) J_{j}(\theta) d \theta=\left\{\begin{array}{l}\frac{-1}{2 s+1} \tilde{I}_{2 k+2 s+2}(2 \pi)= \\ \frac{-\pi \alpha_{k+s+1}}{2^{k+s+1}(2 s+1)(k+s+2) !}, j=2 s, \\ 0, \quad j=2 s+1 .\end{array}\right.$

- $\int_{0}^{2 \pi} T_{2 k+1}(\theta) \tilde{J}_{j}(\theta) d \theta=\left\{\begin{array}{l}-\frac{2}{(2 s+1)} \tilde{I}_{2 k+2 s+2}(2 \pi)+\frac{2}{(2 s+3)} \tilde{I}_{2 k+2 s+4}(2 \pi)= \\ \frac{-\pi(4 k+10 s+15) \alpha_{k+s+1}}{2^{k+s+2}(2 s+1)(2 s+3)(k+s+3) !}, j=2 s, \\ 0, \quad j=2 s+1 .\end{array}\right.$

- $\int_{0}^{2 \pi} R_{i}(\theta) \sin (2 l+1) \theta d \theta=\pi C_{i, l}$.

- $\int_{0}^{2 \pi} S_{i}(\theta) \sin (2 l+1) \theta d \theta=\pi K_{i, l}$. 
Hence Lemma 1 is proved.

Lemma 4. The integral $F_{20}^{2}(r)$ is a polynomial in the variable $r$ is

$$
\begin{aligned}
F_{20}^{2}(r)= & \sum_{s=0}^{\left[\frac{n}{2}\right]} \frac{\alpha_{s}}{2^{s+1}(s+1) !} q_{2 s} r^{2 s+1}-\sum_{s=0}^{\left[\frac{n}{2}\right]} \sum_{k=0}^{\left[\frac{n-1}{2}\right]} r^{2 s+2 k+1}\left(a_{2 s} b_{2 k+1} \frac{\alpha_{s+k+1}}{2^{s+k+1}(s+k+2) !}\right. \\
& \left.+c_{2 s} b_{2 k+1} r^{2} \frac{3 \alpha_{s+k+1}}{2^{s+k+2}(s+k+3) !}\right)-\sum_{k=0}^{\left[\frac{n-1}{2}\right]\left[\frac{m-1}{2}\right]} \sum_{s=0}^{2} e_{2 s+1} b_{2 k+1} r^{2 s+2 k+3} \\
& \times \frac{(s+k) \alpha_{s+k+1}}{2^{s+k+2}(s+k+3) !} .
\end{aligned}
$$

Proof. Using (4.5) and, substituting in (4.4) we have

$$
\begin{aligned}
F_{2}(\theta, r)= & \sum_{i=0}^{n} r^{i}\left(p_{i} R_{i}(\theta)+q_{i} r T_{i}(\theta)+s_{i} r^{2} S_{i}(\theta)\right)+\sum_{i=0}^{m} v_{i} r^{i+1} R_{i+1}(\theta)-\frac{1}{r}\left(\sum _ { i = 0 } ^ { n } r ^ { i } \left(a_{i}\right.\right. \\
& \left.\left.\times R_{i}(\theta)+c_{i} r^{2} S_{i}(\theta)\right)+\sum_{k=0}^{\left[\frac{n-1}{2}\right]} r^{2 k+2} b_{2 k+1} T_{2 k+1}(\theta)+\sum_{i=0}^{m} e_{i} r^{i+1} R_{i+1}(\theta)\right) \\
& \times\left(\sum_{i=0}^{n} r^{i}\left(a_{i} \cos ^{i+1}(\theta)+c_{i} r^{2} T_{i+1}(\theta)\right)+\sum_{k=0}^{\left[\frac{n-1}{2}\right]} r^{2 k+2} b_{2 k+1} R_{2 k+2}(\theta)\right. \\
& \left.-\sum_{i=0}^{m} e_{i} r^{i+1} T_{i}(\theta)\right),
\end{aligned}
$$

then

$$
\begin{aligned}
F_{20}^{2}(r)= & \sum_{i=0}^{n} r^{i}\left(p_{i} \int_{0}^{2 \pi} R_{i}(\theta) d \theta+q_{i} r \int_{0}^{2 \pi} T_{i}(\theta) d \theta+s_{i} r^{2} \int_{0}^{2 \pi} S_{i}(\theta) d \theta\right) \\
& -\sum_{i=0}^{n} \sum_{j=0}^{n} r^{i+j-1}\left(a_{i} a_{j} \int_{0}^{2 \pi} R_{i}(\theta) \cos ^{j+1} \theta d \theta+a_{i} c_{j} r^{2} \int_{0}^{2 \pi} R_{i}(\theta) T_{j+1}(\theta) d \theta\right. \\
& \left.+c_{i} a_{j} r^{2} \int_{0}^{2 \pi} S_{i}(\theta) \cos ^{j+1} \theta d \theta+c_{i} c_{j} r^{4} \int_{0}^{2 \pi} S_{i}(\theta) T_{j+1}(\theta) d \theta\right)-\sum_{i=0}^{n} \sum_{k=0}^{\left[\frac{n-1}{2}\right]} r^{i+2 k+1} \\
& \times\left(a_{i} b_{2 k+1}\left(\int_{0}^{2 \pi} T_{2 k+1}(\theta) \cos ^{i+1} \theta d \theta+\int_{0}^{2 \pi} R_{2 k+2}(\theta) R_{i}(\theta) d \theta\right)+c_{i} b_{2 k+1} r^{2}\right. \\
& \left.\times\left(\int_{0}^{2 \pi} T_{2 k+1}(\theta) T_{i+1}(\theta) d \theta+\int_{0}^{2 \pi} R_{2 k+2}(\theta) S_{i}(\theta) d \theta\right)\right)+\sum_{i=0}^{n} \sum_{j=0}^{m} r^{i+j}\left(a_{i} e_{j}\right. \\
& \times\left(\int_{0}^{2 \pi} R_{i}(\theta) T_{j}(\theta) d \theta-\int_{0}^{2 \pi} R_{j+1}(\theta) \cos ^{i+1} \theta d \theta\right)+c_{i} e_{j} r^{2}\left(\int_{0}^{2 \pi} S_{i}(\theta) T_{j}(\theta) d \theta\right.
\end{aligned}
$$




$$
\begin{aligned}
& \left.\left.-\int_{0}^{2 \pi} R_{j+1}(\theta) T_{i+1}(\theta) d \theta\right)\right)+\sum_{s=0}^{\left[\frac{n-1}{2}\right]\left[\frac{n-1}{2}\right]} \sum_{k=0} b_{2 s+1} b_{2 k+1} r^{2 s+2 k+3} \int_{0}^{2 \pi} T_{2 s+1}(\theta) \\
& \times R_{2 k+2}(\theta) d \theta+\sum_{k=0}^{\left[\frac{n-1}{2}\right]} \sum_{i=0}^{m} e_{i} b_{2 k+1} r^{i+2 k+2}\left(-\int_{0}^{2 \pi} R_{2 k+2}(\theta) R_{i+1}(\theta) d \theta\right) \\
& +\int_{0}^{2 \pi} T_{i}(\theta) T_{2 k+1}(\theta) d \theta+\sum_{i=0}^{m} \sum_{j=0}^{m} e_{i} e_{j} r^{i+j+1} \int_{0}^{2 \pi} R_{j+1}(\theta) T_{i}(\theta) d \theta
\end{aligned}
$$

For an explicit expression of the polynomial $F_{20}^{2}(r)$, using the integrals of appendix A we have

- $\int_{0}^{2 \pi} R_{i}(\theta) d \theta=\int_{0}^{2 \pi} S_{i}(\theta) d \theta=\int_{0}^{2 \pi} \cos ^{i+1} \theta R_{j}(\theta) d \theta=\int_{0}^{2 \pi} \cos ^{i+1} \theta S_{j}(\theta) d \theta=$ $\int_{0}^{2 \pi} R_{i}(\theta) T_{j+1}(\theta) d \theta=\int_{0}^{2 \pi} S_{i}(\theta) T_{j+1} d \theta=0$.

- $\int_{0}^{2 \pi} T_{i}(\theta) d \theta= \begin{cases}0, & \text { if } i=2 k+1, \\ \tilde{I}_{2 k}(2 \pi)=\frac{\pi \alpha_{k}}{2^{k}(k+1) !}, & \text { if } i=2 k .\end{cases}$

- $\int_{0}^{2 \pi} \cos ^{i+1} \theta T_{2 k+1}(\theta) d \theta=\int_{0}^{2 \pi} R_{2 k+2}(\theta) R_{i}(\theta) d \theta=$

$\begin{cases}0, & \text { if } i=2 s+1, \\ \tilde{I}_{2 k+2 s+2}(2 \pi)=\frac{\pi \alpha_{k+s+1}}{2^{k+s+1}(k+s+2) !}, & \text { if } i=2 s .\end{cases}$

- $\int_{0}^{2 \pi} R_{2 k+2}(\theta) S_{i}(\theta) d \theta=\int_{0}^{2 \pi} T_{2 k+1}(\theta) T_{i+1}(\theta) d \theta$

$= \begin{cases}0, & \text { if } i=2 s+1, \\ \tilde{I}_{2 k+2 s+2}(2 \pi)-\tilde{I}_{2 k+2 s+4}(2 \pi)=\frac{3 \pi \alpha_{k+s+1}}{2^{k+s+2}(k+s+3) !}, & \text { if } i=2 s .\end{cases}$

- $\int_{0}^{2 \pi} T_{2 k+1}(\theta) T_{i}(\theta) d \theta= \begin{cases}0, & \text { if } i=2 s, \\ \tilde{I}_{2 k+2 s+2}(2 \pi)-\tilde{I}_{2 k+2 s+4}(2 \pi) & \text { if } i=2 s+1 . \\ =\frac{3 \pi \alpha_{k+s+1}}{2^{k+s+2}(k+s+3) !}, & \end{cases}$

- $\int_{0}^{2 \pi} R_{2 k+2}(\theta) R_{i+1}(\theta) d \theta= \begin{cases}0, & \text { if } i=2 s, \\ \tilde{I}_{2 k+2 s+4}(2 \pi)=\frac{\pi \alpha_{k+s+2}}{2^{k+s+2}(k+s+3) !}, & \text { if } i=2 s+1 .\end{cases}$

Hence Lemma 2 is proved.

By Lemma 1 and Lemma 2, we have

$$
\begin{aligned}
& F_{20}(r)=F_{20}^{1}(r)+F_{20}^{2}(r) \\
& =\sum_{s=0}^{\left[\frac{n}{2}\right]} r^{2 s+1} \frac{\alpha_{s}}{2^{s+1}(s+1) !} q_{2 s}+\sum_{s=0}^{\left[\frac{n}{2}\right]} \sum_{k=0}^{\left[\frac{n-1}{2}\right]} r^{2 s+2 k+1}\left(a _ { 2 s } b _ { 2 k + 1 } \left(s \sum_{l=0}^{k+1} \tilde{\gamma}_{k, l} C_{s, l}\right.\right. \\
& \left.-\frac{(2 s+k+2) \alpha_{k+s+1}}{2^{k+s+1}(2 s+1)(k+s+2) !}\right)+c_{2 s} b_{2 k+1} r^{2}\left((s+1) \sum_{l=0}^{k+1} \tilde{\gamma}_{k, l} K_{s, l}\right. \\
& -\frac{\left.\left(12 s^{2}+4 k^{2}+34 s+10 s k+19 k+24\right) \alpha_{k+s+1}\right)}{2^{k+s+2}(2 s+1)(2 s+3)(k+s+3) !}+\sum_{k=0}^{\left[\frac{n-1}{2}\right]} \sum_{s=0}^{\left[\frac{m-1}{2}\right]} r^{2 s+2 k+3}
\end{aligned}
$$




$$
\begin{aligned}
& \times e_{2 s+1} b_{2 k+1}\left(-\frac{\left(2 s^{2}+5 s+4 s k+8 k+2 k^{2}+3\right) \alpha_{k+s+1}}{2^{k+s+2}(2 s+3)(k+s+3) !}+(s+1)\right. \\
& \left.\times \sum_{l=0}^{k+1} \tilde{\gamma}_{k, l} C_{s+1, l}\right) .
\end{aligned}
$$

Then, to find the real positive roots of $F_{20}(r)$, we must find the zeros of a polynomial in $r^{2}$ of degree equal to the $\max \left\{\left[\frac{n}{2}\right]+\left[\frac{n-1}{2}\right]+1,\left[\frac{n-1}{2}\right]+\left[\frac{m-1}{2}\right]+1\right\}$. Since $\left[\frac{n-1}{2}\right]+\left[\frac{n}{2}\right]+1$ takes the values $\left[\frac{n-1}{2}\right]+\left[\frac{n-1}{2}\right]+1$ if $n$ is odd, and the $\left[\frac{n}{2}\right]+\left[\frac{n-2}{2}\right]+1$ if $n$ is even, that is $n$ in both cases. We conclude that $F_{20}(r)$ has at most $\max \left\{n,\left[\frac{n-1}{2}\right]+\left[\frac{m-1}{2}\right]+1\right\}$ simple positive zeros.

Example. In this Example we prove that the upper bounds $\tilde{H}_{2}(m, n)$ is reached in the case when $1 \leq n \leq 5$ and $1 \leq m \leq 5$. The computations have been verified with the help of Maple. We denote by $F_{20}^{m, n}(r)$ the function $F_{20}(r) / r$ corresponding, an adequate computation allows to obtain that

- $F_{20}^{m, 1}(r)=\frac{1}{2} q_{0}-\frac{1}{2} a_{0} b_{1}-\frac{1}{8} c_{0} b_{1} r^{2}$, now if we fixed $a_{0}=\frac{1}{2}, b_{1}=-2, c_{0}=-4$ and $q_{0}=1$, we have that $F_{20}^{m, 1}(r)=1-r^{2}$, that has exactly one positive zero.

- $F_{20}^{m, 2}(r)=\frac{1}{2} q_{0}-\frac{1}{2} a_{0} b_{1}+\left(\frac{1}{8} q_{2}-\frac{1}{8} c_{0} b_{1}-\frac{1}{8} a_{2} b_{1}\right) r^{2}-\frac{1}{48} b_{1} c_{2} r^{4}$, now if we fixed $a_{0}=\frac{1}{2}, a_{2}=-3$, $b_{1}=-2, c_{0}=-4, c_{2}=12, q_{0}=1$ and $q_{2}=2$, we have that $F_{20}^{m, 2}(r)=\frac{1}{2}\left(1-r^{2}\right)\left(2-r^{2}\right)$, that has exactly two positive zeros.

- $F_{20}^{1,3}(r)=F_{20}^{2,3}(r)=\frac{1}{2} q_{0}-\frac{1}{2} a_{0} b_{1}+\left(\frac{1}{8} q_{2}-\frac{1}{8} c_{0} b_{1}-\frac{1}{8} a_{2} b_{1}-\frac{3}{8} a_{0} b_{3}\right) r^{2}+\left(-\frac{1}{24} e_{1} b_{3}-\frac{1}{48} b_{1} c_{2}-\right.$ $\left.\frac{5}{48} a_{2} b_{3}-\frac{7}{48} c_{0} b_{3}\right) r^{4}-\frac{3}{128} c_{2} b_{3} r^{6}$, now if we fixed $a_{0}=\frac{1}{2}, a_{2}=-\frac{35}{9}, b_{1}=-2, b_{3}=\frac{16}{27}, c_{0}=-4$, $c_{2}=12, q_{0}=1, q_{2}=2$ and $e_{1}=\frac{125}{36}$, we have that $F_{20}^{1,3}(r)=F_{20}^{2,3}(r)=\frac{1}{6}\left(1-r^{2}\right)\left(2-r^{2}\right)\left(3-r^{2}\right)$, that has exactly three positive zeros.

- $F_{20}^{3,3}(r)=F_{20}^{4,3}(r)=F_{20}^{1,3}(r)-\frac{1}{64} e_{3} b_{3} r^{6}$, now if we fixed $a_{0}=\frac{1}{2}, a_{2}=-3, b_{1}=-2, b_{3}=\frac{16}{9}$, $c_{0}=-4, c_{2}=12, q_{0}=1, q_{2}=2, e_{1}=\frac{59}{4}$ and $e_{3}=-12$, we have that $F_{20}^{3,3}(r)=F_{20}^{4,3}(r)=$ $\frac{1}{6}\left(1-r^{2}\right)\left(2-r^{2}\right)\left(3-r^{2}\right)$, that has exactly three positive zeros.

- $F_{20}^{5,3}(r)=F_{20}^{3,3}(r)-\frac{1}{128} b_{3} e_{5} r^{8}$, now if we fixed $a_{0}=\frac{1}{2}, a_{2}=-3, b_{1}=-2, b_{3}=\frac{28}{9}, c_{0}=-4$, $c_{2}=12, q_{0}=1, q_{2}=2, e_{1}=\frac{395}{28}, e_{3}=-\frac{66}{7}$ and $e_{5}=-\frac{12}{7}$, we have that $F_{20}^{5,3}(r)=$ $\frac{1}{24}\left(1-r^{2}\right)\left(2-r^{2}\right)\left(3-r^{2}\right)\left(4-r^{2}\right)$, that has exactly four positive zeros.

- $F_{20}^{1,4}(r)=F_{20}^{2,4}(r)=\frac{1}{2} q_{0}-\frac{1}{2} a_{0} b_{1}+\left(\frac{1}{8} q_{2}-\frac{1}{8} c_{0} b_{1}-\frac{1}{8} a_{2} b_{1}-\frac{3}{8} a_{0} b_{3}\right) r^{2}+\left(\frac{1}{16} q_{4}-\frac{1}{24} e_{1} b_{3}-\frac{1}{48} b_{1} c_{2}-\right.$ $\left.\frac{5}{48} a_{2} b_{3}-\frac{7}{48} c_{0} b_{3}-\frac{1}{16} a_{4} b_{1}\right) r^{4}+\left(-\frac{3}{128} c_{2} b_{3}-\frac{1}{128} b_{1} c_{4}-\frac{7}{128} a_{4} b_{3}\right) r^{6}-\frac{11}{1280} b_{3} c_{4} r^{8}$, now if we fixed $a_{0}=\frac{1}{2}, a_{2}=-\frac{44}{9}, a_{4}=\frac{1161}{308}, b_{1}=-2, b_{3}=\frac{16}{27}, c_{0}=-4, c_{2}=12, c_{4}=-\frac{90}{11}, q_{0}=1, q_{2}=2$, $q_{4}=-2$ and $e_{1}=\frac{63433}{44352}$ we have that $F_{20}^{1,4}(r)=F_{20}^{2,4}(r)=\frac{1}{24}\left(1-r^{2}\right)\left(2-r^{2}\right)\left(3-r^{2}\right)\left(4-r^{2}\right)$, that 
has exactly four positive zeros.

- $F_{20}^{3,4}(r)=F_{20}^{4,4}(r)=F_{20}^{1,4}(r)-\frac{1}{64} e_{3} b_{3} r^{6}$, now if we fixed $a_{0}=\frac{1}{2}, a_{2}=-3, a_{4}=\frac{657}{308}, b_{1}=-2$, $b_{3}=\frac{16}{9}, c_{0}=-4, c_{2}=12, c_{4}=-\frac{30}{11}, q_{0}=1, q_{2}=0, q_{4}=\frac{1417}{462}, e_{1}=\frac{59}{4}$ and $e_{3}=-12$ we have that $F_{20}^{3,4}(r)=F_{20}^{4,4}(r)=\frac{1}{24}\left(1-r^{2}\right)\left(2-r^{2}\right)\left(3-r^{2}\right)\left(4-r^{2}\right)$, that has exactly four positive zeros.

- $F_{20}^{5,4}(r)=F_{20}^{3,4}(r)-\frac{1}{128} b_{3} e_{5} r^{8}$, now if we fixed $a_{0}=\frac{1}{2}, a_{2}=-3, a_{4}=\frac{1}{2}, b_{1}=-2, b_{3}=\frac{28}{9}$, $c_{0}=-4, c_{2}=12, c_{4}=\frac{49}{9}, q_{0}=1, q_{2}=2, q_{4}=-1, e_{1}=\frac{395}{28}, e_{3}=-\frac{66}{7}$ and $e_{5}=-\frac{4853}{630}$ we have that $F_{20}^{5,4}(r)=\frac{1}{24}\left(1-r^{2}\right)\left(2-r^{2}\right)\left(3-r^{2}\right)\left(4-r^{2}\right)$, that has exactly four positive zeros.

- $F_{20}^{1,5}(r)=F_{20}^{2,5}(r)=\frac{1}{2} q_{0}-\frac{1}{2} a_{0} b_{1}+\left(\frac{1}{8} q_{2}-\frac{1}{8} c_{0} b_{1}-\frac{1}{8} a_{2} b_{1}-\frac{3}{8} a_{0} b_{3}\right) r^{2}+\left(\frac{1}{16} q_{4}-\frac{1}{24} e_{1} b_{3}-\frac{1}{48} b_{1} c_{2}-\right.$ $\left.\frac{5}{48} a_{2} b_{3}-\frac{7}{48} c_{0} b_{3}-\frac{1}{16} a_{4} b_{1}-\frac{5}{16} a_{0} b_{5}\right) r^{4}+\left(-\frac{3}{128} c_{2} b_{3}-\frac{1}{128} b_{1} c_{4}-\frac{7}{128} a_{4} b_{3}-\frac{5}{96} b_{5} e_{1}-\frac{35}{384} a_{2} b_{5}-\frac{55}{384} c_{0} b_{5}\right) r^{6}+$ $\left(-\frac{63}{1280} a_{4} b_{5}-\frac{91}{3840} c_{2} b_{5}-\frac{11}{1280} b_{3} c_{4}\right) r^{8}-\frac{9}{1024} c_{4} b_{5} r^{10}$, now if we fixed $a_{0}=\frac{1}{2}, a_{2}=-\frac{1410989}{213444}, a_{4}=$ $\frac{6121}{693}, b_{1}=-2, b_{3}=\frac{16}{27}, b_{5}=-\frac{704}{6075}, c_{0}=-4, c_{2}=12, c_{4}=-\frac{90}{11} q_{0}=1, q_{2}=\frac{683623}{177870}$, $q_{4}=-\frac{35777327}{4802490}$ and $e_{1}=\frac{63433}{44352}$ we have that $F_{20}^{1,5}(r)=F_{20}^{2,5}(r)=\frac{1}{120}\left(1-r^{2}\right)\left(2-r^{2}\right)\left(3-r^{2}\right)(4-$ $\left.r^{2}\right)\left(5-r^{2}\right)$, that has exactly five positive zeros.

- $F_{20}^{3,5}(r)=F_{20}^{4,5}(r)=F_{20}^{1,5}(r)-\frac{1}{64} b_{3} e_{3} r^{6}-\frac{7}{320} b_{5} e_{3} r^{8}$, now if we fixed $a_{0}=\frac{1}{2}, a_{2}=-\frac{596927}{30492}$, $a_{4}=-3, b_{1}=-2, b_{3}=\frac{16}{27}, b_{5}=-\frac{704}{6075}, c_{0}=-4, c_{2}=12, c_{4}=-\frac{90}{11}, q_{0}=1, q_{2}=\frac{756589}{25410}$, $q_{4}=\frac{334177}{98010}, e_{1}=\frac{63433}{44352}$ and $e_{3}=\frac{2050}{77}$ we have that $F_{20}^{3,5}(r)=F_{20}^{4,5}(r)=\frac{1}{120}\left(1-r^{2}\right)\left(2-r^{2}\right)(3-$ $\left.r^{2}\right)\left(4-r^{2}\right)\left(5-r^{2}\right)$, that has exactly five positive zeros.

- $F_{20}^{5,5}(r)=F_{20}^{3,5}(r)-\frac{1}{128} b_{3} e_{5} r^{8}-\frac{3}{256} b_{5} e_{5} r^{10}$, now if we fixed $a_{0}=\frac{1}{2}, a_{2}=-3, a_{4}=\frac{1}{2}, b_{1}=-2$, $b_{3}=\frac{28}{9}, b_{5}=1, c_{0}=-4, c_{2}=12, c_{4}=\frac{49}{9}, q_{0}=1, q_{2}=\frac{2}{5}, q_{4}=\frac{1093657}{21870}, e_{1}=\frac{27767}{810}, e_{3}=-\frac{34393}{1512}$ and $e_{5}=-\frac{607}{180}$ we have that $F_{20}^{5,5}(r)=\frac{1}{120}\left(1-r^{2}\right)\left(2-r^{2}\right)\left(3-r^{2}\right)\left(4-r^{2}\right)\left(5-r^{2}\right)$, that has exactly five positive zeros.

\section{Appendix A. Formulae}

In this appendix we recall some formulae that will be used during the paper, see for more details [1]. For $i \geq 0$ we have

$$
\begin{aligned}
& J_{i}(\theta)=\int_{0}^{\theta} \cos ^{i} t \sin t d t=\frac{1}{i+1}\left(1-\cos ^{i+1} \theta\right) . \\
& \tilde{J}_{i}(\theta)=\int_{0}^{\theta} \cos ^{i} t \sin ^{3} t d t=\frac{2}{(i+1)(i+3)}-\frac{1}{i+1} \cos ^{i+1} \theta+\frac{1}{i+3} \cos ^{i+3} \theta . \\
& J_{i}(2 \pi)=\tilde{J}_{i}(2 \pi)=0 .
\end{aligned}
$$


$I_{i}(\theta)=\int_{0}^{2 \pi} \cos ^{i} \theta d \theta= \begin{cases}\sum_{l=0}^{k} \gamma_{k, l} \sin (2 l+1) \theta, & \text { if } \mathrm{i}=2 \mathrm{k}+1 ; \\ \delta_{k}+\sum_{l=1}^{k} \beta_{k, l} \sin (2 l \theta), & \text { if } \mathrm{i}=2 \mathrm{k},\end{cases}$

where

$$
\delta_{i}=\frac{1}{2^{2 i}}\left(\begin{array}{c}
2 i \\
i
\end{array}\right) \theta, \quad \gamma_{i, l}=\frac{1}{2^{2 i}}\left(\begin{array}{c}
2 i+1 \\
i-l
\end{array}\right) \frac{1}{2 l+1}, \quad \beta_{i, l}=\left(\begin{array}{c}
2 i \\
i+l
\end{array}\right) \frac{1}{l}
$$

$I_{i}(2 \pi)= \begin{cases}0, & \text { if } \mathrm{i}=2 \mathrm{k}+1 \\ \frac{{ }^{k-1} k \alpha_{k}}{2^{k-1}}, & \text { if } \mathrm{i}=2 \mathrm{k}\end{cases}$

where

$\alpha_{k}=3.5 \ldots . .(2 k-1), \alpha_{k+1}=(2 k+1) \alpha_{k}$.

And

$I_{2 k+2}(2 \pi)=\frac{2 k+1}{2 k+2} I_{2 k}(2 \pi)$.

$\tilde{I}_{i}(2 \pi)=I_{i}(2 \pi)-I_{i+2}(2 \pi)$.

$\int_{0}^{2 \pi} \cos ^{i} \theta \sin ^{j} \theta \sin (2 l+1) \theta d \theta \neq 0$, if i even and $\mathrm{j}$ odd,

$\int_{0}^{2 \pi} \cos ^{i} \theta \sin ^{j} \theta \sin (2 l+1) \theta d \theta= \begin{cases}0, & \text { if } \mathrm{i} \text { odd } \text { or } \mathrm{j} \text { even; } \\ \pi C_{k, l}, & \mathrm{i}=2 \mathrm{k}, \mathrm{j}=1 \text { and } 1 \geq 0, \\ \pi K_{k, l}, & \mathrm{i}=2 \mathrm{k}, \mathrm{j}=3 \text { and } 1 \geq 0,\end{cases}$

where

$C_{k, l}, K_{k, l}$ are non-zero constants.

\section{References}

[1] M. Abramowitz, I. Stegun, Handbook of Mathematical Functions with Formulas, Graphs, and Mathematical Tables.National Bureau of Standards Applied Mathematics Series, no.55, Washington,DC: US Government Printing Office 1964.

[2] T. R. Blows, G. N. Lloyd, The number of small-amplitude limit cycles of Linard equations. Math. Proc. Camb. Phil. Soc. 1984;95:359-366.

[3] A. Boulfoul, A. Makhlouf and M. Mellahi, On the limit cycles for a class of generalized Kukles differential systems, Journal of Applied Analysis and Computation 2019; 9:864-883.

[4] A. Buica, J. Llibre, Averaging methods for finding periodic orbits via Brouwer degree, Bull. Sci. Math. 2004;128:7-22.

[5] C. J. Christopher, S. Lynch, Limit cycles in highly non-linear differential equations, Journal of Sound and Vibration. 1999;224:505-517.

[6] W. A. Coppel, Some quadratic systems with at most one limit cycles. Dynamics reported, Vol. 2. New York: Wiley; 1998.

[7] P. De Maesschalck, F. Dumortier, Classical Linard equations of degree $n \geq 6$ can have [( $n-1) / 2]$ limit cycles. J Differ Equ 2011;250:2162-76.

[8] F. Dumortier, C. Li, On the uniqueness of limit cycles surrounding one or more singularities for Linard equations. Nonlinearity 1996;9:1489-500. 
[9] F. Dumortier, C. Li, Quadratic Linard equations with quadratic damping. J Differ Equ 1997; 139:41-59.

[10] F. Dumortier, D. Panazzolo, R. Roussarie, More limit cycles than expected in Linard systems. Proc Am Math Soc 2007;135:1895-904.

[11] F. Dumortier, C. Rousseau, Cubic Linard equations with linear dapimg. Nonlinearity 1990; 3:1015-1039.

[12] B. Garca, J. Llibre, J. S. Prez del Ro, Limit cycles of generalized Linard polynomial differential systems via averaging theory. Chaos, Solitons Fractals 2014;62-63:1-9.

[13] A. Gasull, J. Torregrosa, Samll-amplitude limit cycles in Linard systems via multiplicity. J Differ Equ 1998;159:1015-1039.

[14] D. Hilbert,Mathematische probleme, em lecture in: secondInternat. Cong. Math, Paris, 1900, Nachr. Ges. Wiss. Gttingen. Math. Phys. Ki 5 (1900), 253-297; English Transl: Bull. Amer. Math. Soc. 1902;8:437-479.

[15] C. Li, J. Llibre, Uniqueness of limit cycles for Linard differential equations of degree four. J Differ Equ 2012;252:314262.

[16] A. Linard, tude des oscillations entrenues. Revue gnrale de l'lectricit 1928;23:946-954.

[17] A. Lins, W. de Melo, C. C. Pugh, On Linard's equation, Lecture notes in Math Nonlinear 597, Springer, 1977;pp:335-357.

[18] J. Llibre, A. C. Mereu, M.A Teixeira, Limit cycles of the generalized polynomial Linard differential equations. Math Proc Camb Phil Soc 2010;148:363-383.

[19] J. Llibre, C. Valls, Limit cycles for a generalization of Linard polynomial differential systems. Chaos Solitons Fractals 2013;46:65-74.

[20] J. Llibre, C. Valls, On the number of limit cycles for a generalization of Linard polynomial differential systems. Int J Bifurcation Chaos 2013;23 1350048-16.

[21] J. Llibre, C. Valls, On the number of limit cycles of a class of polynomial differential systems. Proc A R Soc 2012;468:2347-2360.

[22] G. N. Lloyd, Limit cycles of polynomial systems-some recent developments. London Math. Soc. Lecture note Ser. 127, Cambridge University Press 1988;PP:192-234.

[23] N. G. Lloyd, S. Lynch, Small-amplitude Limit cycles of certain Linard systems, Proc. Royal Soc. Proc R Soc Lond Ser A 1988;418:199-208.

[24] S. Lynch, Limit cycles of generalized Linard equations. Appl. Math. Lett. 1995;8:15-17.

[25] N. Mellahi, A. Boulfoul and A. Makhlouf, Maximum number of limit cycles for generalized Kukles polynomial differential systems, Diff. Equ. Dyn. Syst $2019 ; 27(4): 493-514$.

[26] G. S. Rychkov, The maximum number of limit cycle of the system $\dot{x}=y-a_{1} x^{3}-a_{2} x^{5}, \dot{y}=-x$ is two. Differ Uravn 1975;11:380-391.

[27] J. A. Sanders, F. Verhust, Averaging Methods in Nonlinear Dynamical Systems, Applied Mathematical Sciences.59 Springer-Verlag, New York; 1985.

[28] S. Smale, Mathematical problems for the next century. Math. Intelligencer 1998;20:7-15.

[29] F. Verhulst, Nonlinear Differential Equations and Dynamical Systems, em Berlin: Springer-Verlag, Second Edition; 1991.

[30] P. Yu, M. Han, Limit cycles in generalized Linard systems. Chaos Solitons Fract 2006;30:1048-1068. 